\title{
A Pilot Study to Assess the Feasibility of a Web-Based Survey to Examine Patient-Reported Symptoms and Satisfaction in Patients with Ankylosing Spondylitis Receiving Secukinumab
}

\author{
Marina Magrey ${ }^{1} \cdot$ Michael Bozyczko $^{2} \cdot$ Daniel Wolin $^{3} \cdot$ Margaret Mordin $^{3} \cdot$ Lori McLeod $^{3} \cdot$ Eric Davenport $^{3}$. \\ Costel Chirila ${ }^{3} \cdot$ Yujin Park ${ }^{4}$
}

Published online: 3 May 2019

(c) The Author(s) 2019

\begin{abstract}
Purpose This real-world study evaluated the feasibility of assessing patient-reported symptom improvement and treatment satisfaction using a web-based survey among patients with ankylosing spondylitis (AS) treated with secukinumab.

Methods This cross-sectional, web-based survey collected data on demographics, symptoms, treatment history, and treatment satisfaction from US patients with AS who were receiving secukinumab at survey participation. Patients reported AS symptoms experienced before and after secukinumab initiation, time to symptom improvement, and satisfaction with secukinumab treatment.

Results Of 2755 patients screened, 200 with AS were included in the analysis. The mean (SD) age of patients was 34.4 (10.6) years; $86.5 \%$ were biologic experienced. Most (74.0\%) reported overall improvement ("a little," "moderately," or "much better") in AS symptoms since secukinumab initiation compared with before secukinumab initiation; a similar trend was observed for all the individual symptoms analyzed (pain disrupting sleep, fatigue, morning stiffness, pain and stiffness in lower back or neck, sore areas other than joints, and ankle or heel pain [indicating enthesitis]). Approximately $41.9 \%$ of patients reported overall symptom improvement within 4 weeks of secukinumab treatment. Most expressed overall satisfaction ("very," "mostly," or "somewhat satisfied") with secukinumab regarding symptom improvement (99.0\%), speed of symptom improvement (97.0\%), frequency and method of administration (96.0\% and $91.5 \%$, respectively), ease of use (93.5\%), patient support services (97.0\%), and side effects, if any (93.0\%).

Conclusion Most patients reported overall symptom improvement and satisfaction with treatment. Our study indicates that patient-reported perspectives may be feasibly collected using a web-based survey to provide insights into treatment experience and satisfaction.
\end{abstract}

Electronic supplementary material The online version of this article (https://doi.org/10.1007/s40801-019-0154-4) contains supplementary material, which is available to authorized users.

Marina Magrey

mmagrey@metrohealth.org

1 Division of Rheumatology, The MetroHealth System and School of Medicine, Case Western Reserve University, 2500 MetroHealth Dr., Cleveland, OH 44109, USA

2 National Psoriasis Foundation, Portland, OR, USA

3 RTI Health Solutions, Research Triangle Park, NC, USA

4 Novartis Pharmaceuticals Corporation, East Hanover, NJ, USA

\section{Key Points}

We explored the feasibility of assessing real-world patient experiences regarding their overall treatment experience for ankylosing spondylitis (AS) using simple questions in plain language to assess their treatment experience and satisfaction; in this study, their experience with secukinumab was evaluated.

The majority of respondents reported AS symptom improvement, which was achieved within 6 months, and satisfaction with secukinumab.

Our data indicate that assessing patient-reported perspectives of patients with AS using a web-based survey may be a practical means to provide insights into treatment experience and satisfaction. 


\section{Introduction}

Ankylosing spondylitis (AS) is a chronic, inflammatory rheumatic disease that predominantly affects the axial skeleton, resulting in bony ankylosis and often leading to impairment or loss of physical function [1-3]. Patients with AS are known to have a higher risk for many co-morbid conditions, including cardiovascular disease and osteoporosis [4-9]. AS is associated with decreased quality of life (QOL), increased mortality, and substantial healthcare-related costs, therefore imposing a high burden on patients, their caregivers, and society [10].

Based on recommendations from the Assessment of SpondyloArthritis international Society/European League Against Rheumatism [11], American College of Rheumatology, Spondylitis Association of America, and Spondyloarthritis Research and Treatment Network [12], the first-line treatment for AS should include nonsteroidal antiinflammatory drugs (NSAIDs) for the management of pain and stiffness. For patients with an inadequate response to NSAIDs, or who experience adverse effects, biologics have emerged as a novel anti-inflammatory therapy [13]. Current practice suggests tumor necrosis factor inhibitors as first-line biologic therapy for patients with persistent disease activity despite NSAID treatment [14].

Significant progress has been made in our understanding of AS in the last decade, and interleukin (IL)-17 has been identified as a key cytokine driving inflammation in AS [15-17]. Several studies suggest that the IL-17 pathway is a promising target in the treatment of AS [18-20]. Additionally, patients with AS have elevated numbers of IL17-producing cells [21-23]. Secukinumab, a fully human monoclonal antibody targeting IL-17A, is the first and only approved non-TNFi biologic approved in the USA for the treatment of AS; it is also indicated for the treatment of psoriasis and psoriatic arthritis (PsA) [24].

In two phase III placebo-controlled trials (MEASURE 1 and MEASURE 2), secukinumab showed efficacy in reducing the signs and symptoms of AS at week 16 following treatment initiation; benefits were sustained through week 52 [25, 26]. Based on MEASURE 1 and MEASURE 2, secukinumab was approved in the USA for the treatment of active AS [27]. Even though secukinumab has demonstrated efficacy in randomized clinical trials, little is known about patient experience with secukinumab for the treatment of AS in real-world settings. Disease activity measures, such as the Bath Ankylosing Spondylitis Disease Activity Index (BASDAI) and Assessment of SpondyloArthritis international Society criteria, are routinely used to measure treatment efficacy in clinical trials and are recommended for disease assessment in clinical practice [28]. However, these composite disease activity measures do not fully capture patient perspectives on treatment effectiveness. Additionally, randomized clinical trials often do not account for patient experience and satisfaction with treatment.

In this exploratory study, we sought to determine the feasibility of garnering and understanding real-world patient narratives with regard to their treatment experience and satisfaction. We surveyed patients with active AS who were receiving treatment with secukinumab and collected their accounts of AS symptom improvement and treatment satisfaction in a real-world setting in the USA. Our overarching goal is to develop a practical means of capturing treatmentrelated experiences from the patient perspective.

\section{Methods}

\subsection{Study Design and Patient Population}

Data were collected from a cross-sectional, web-based survey in the USA. A random sample of patients with AS or PsA were invited by email to participate in an online survey hosted by Survey Sampling International (SSI) through their patient panels of self-reported diagnosis of AS or PsA. All panel members were recruited following a double optin process. Patients were fully profiled and pre-identified as having the disease of interest. They answered a series of questions to confirm that they met the inclusion criteria for the study using a screener module of the survey, which was completed prior to the full survey. For patients with AS, eligibility criteria included the ability to read and sign an informed consent form, age $\geq 18$ years, self-reported diagnosis of active AS, treatment with secukinumab at the time of survey participation and continuous treatment since initiation, and willingness to complete the online survey. A separate survey for patients with PsA was administered to those meeting the eligibility criteria for PsA; results of the PsA analysis are reported elsewhere.

SSI uses a variety of contact methods to recruit online panels. Participants for this study were recruited through many channels, including partnerships with trusted loyalty programs, online banner advertisements, TV advertising, emails, apps, social media influencers, websites, and offline methods. SSI provided end-to-end operational services for the patient surveys, and was responsible for programming, hosting, and data collection for the web-based surveys. SSI was also responsible for questionnaire review, communication of online best practices, online survey testing and quality control, oversight of data collection and field update summaries, as well as review and quality control of data files and deliverables. RTI-HS managed the survey administration using the SSI data collection system. SSI developed and hosted the system on a secure server and RTI-HS performed user acceptance testing of the system. Patient 
data were entered directly into the SSI web-based survey platform. Contactable personal identifiers (including email address) were not stored with patient data, and study data were deidentified prior to exporting to RTI-HS.

Data management activities included the development and approval of the data management plan, all annotated data collection forms, data cleaning specifications document, and the testing of summary reports before the system was used live in the field. RTI-HS uses standard operating procedures to program, test, and manage hosting of the data system. User acceptance testing was conducted to ensure that the system met the requirements of the study. Data cleaning checks appeared in real time to alert the patients to missing and out-of-range data.

Each AS survey invitation included a unique survey link, embedded password for access to the survey, and a unique random-user identification number to ensure that each eligible participant completed the questionnaire only once. No personal identifiers were stored with the survey responses, and informed consent was obtained electronically from eligible patients interested in participating in the survey. No patient recruitment was conducted. Thus, approval from institutional review boards was not mandatory.

The survey questionnaire was developed under the guidance of practicing rheumatologist, Dr. Marina Magrey. The questionnaire was subsequently reviewed according to RTI-HS standard operating protocols. Data collected in the survey included demographics, clinical characteristics, co-morbidities, medication history (i.e., never taken, currently taking, taken over the past 12 months), AS symptoms (including pain disrupting sleep, fatigue, morning stiffness, pain and stiffness in the lower back and neck, soreness in areas other than joints, and ankle or heel pain [indicating enthesitis]), disease severity, and experience with secukinumab treatment. Disease activity and functional status at the time of survey participation were evaluated using the Bath Ankylosing Spondylitis Functional Index (BASFI) [29] and BASDAI [30] instruments. Each metric comprises a six- and eight-item questionnaire, respectively, with each question measured on a visual analog scale, resulting in a total score of $0-10$, with higher scores indicating greater severity of disease or impairment of function.

Patients were asked to report their AS symptoms before and after initiating secukinumab treatment, time to symptom improvement, and satisfaction with secukinumab treatment in multiple domains, including symptom improvement, speed of symptom improvement, frequency of treatment, method of administration, ease of use, side effects (if any), and patient support services. With regard to satisfaction, patients were asked to denote whether they were "very satisfied," "mostly satisfied," "somewhat satisfied," or "not satisfied" with these domains; patients were considered satisfied if they denoted "very," "mostly," or "somewhat satisfied."

\subsection{Data Analysis}

Descriptive analyses of patient demographics, self-reported disease severity, treatment history, AS symptoms, and treatment experience were conducted for all patients with AS who were included in the study. Mean, SD, median, interquartile range, and range were used to analyze continuousor ordinal-type data. Frequencies and percentages were reported for categorical data. Each question was analyzed individually without any imputation of item-level missing data; no statistical hypothesis testing or sample weighting of questionnaire responses was performed in this study.

\section{Results}

\subsection{Patient Demographics and Clinical Characteristics}

Of 2755 patients screened for participation in both the AS and PsA surveys, 200 eligible patients with AS, including 66 with both PsA and AS, completed the survey and were included in the analysis. Patient demographics and clinical characteristics are described in Table 1. The mean (SD) age of included participants was 34.4 (10.6) years; $60.0 \%$ were male and $66.0 \%$ were White. The mean (SD) time since these patients first noticed symptoms of AS was 5.1 (5.7) years and the mean (SD) time since diagnosis was 3.9 (5.3) years; the mean (SD) age of these patients at the time of AS diagnosis was $30.3(10.8)$ years. Patients were from all regions of the USA (East, 28.0\%; South, 20.0\%; Midwest, $21.5 \%$; West, $17.0 \%$; North, $11.0 \%$; and a proportion of patients preferred not to answer, $2.5 \%$ ); $62.5 \%$ were employed full time. All 200 patients reported one or more co-morbidity, the most common of which were anxiety (39.0\%), PsA (33.0\%), depression (31.5\%), and chronic pain (28.5\%); $81.5 \%$ of patients reported deformities due to AS. Patients had a mean (SD) BASDAI global score of 5.8 (2.25) and a mean (SD) BASFI global score of 5.5 (2.33).

\subsection{Treatment and Medication History}

Of the 200 patients included, 33 (16.5\%) had been receiving secukinumab for AS for 3-4 months, 39 (19.5\%) for 5-6 months, 35 (17.5\%) for 7-9 months, 36 (18.0\%) for 10-12 months, and 57 (28.5\%) for $>12$ months. Table 2 summarizes the current treatment and medication history of patients included in this study. Most patients (98.5\%) had received prior treatment for AS, and of these, equal proportions $(86.5 \%)$ were previously treated with nonbiologics (including NSAIDs, steroids, and physical therapy) and biologics; 27 patients (13.5\%) were biologic naive. Among 
Table 1 Patient demographics and disease characteristics among patients with ankylosing spondylitis (AS) who had been receiving secukinumab for $\geq 3$ months

\begin{tabular}{|c|c|}
\hline Characteristic & $\begin{array}{l}\text { Patients with } \\
\text { AS }(N=200)\end{array}$ \\
\hline Age, mean (SD), year & $34.4(10.6)$ \\
\hline Male, $n(\%)$ & $120(60.0)$ \\
\hline \multicolumn{2}{|l|}{ Race and/or ethnicity, $n(\%)$} \\
\hline White & $132(66.0)$ \\
\hline Hispanic & $36(18.0)$ \\
\hline Black/African American & $40(20.0)$ \\
\hline Other/Prefer not to answer & $22(11.0)$ \\
\hline \multicolumn{2}{|l|}{ Region, $n(\%)$} \\
\hline East & $56(28.0)$ \\
\hline South & $40(20.0)$ \\
\hline Midwest & $43(21.5)$ \\
\hline West & $34(17.0)$ \\
\hline North & $22(11.0)$ \\
\hline Prefer not to answer & $5(2.5)$ \\
\hline \multicolumn{2}{|l|}{ Employment status, $n(\%)$} \\
\hline Full time & $125(62.5)$ \\
\hline Part time & $21(10.5)$ \\
\hline Work at home & $9(4.5)$ \\
\hline Student & $17(8.5)$ \\
\hline Retired & $5(2.5)$ \\
\hline Disabled/unable to work & $22(11.0)$ \\
\hline Prefer not to answer & $1(0.5)$ \\
\hline Time since symptom onset, mean (SD), years & $5.1(5.7)$ \\
\hline Time since diagnosis, mean (SD), years & $3.9(5.3)$ \\
\hline \multicolumn{2}{|l|}{ Current health conditions, $n(\%)^{\mathrm{a}}$} \\
\hline Anxiety & $78(39.0)$ \\
\hline Chronic pain & $57(28.5)$ \\
\hline Depression & $63(31.5)$ \\
\hline Fatigue & $64(32.0)$ \\
\hline Psoriatic arthritis & $66(33.0)$ \\
\hline BASDAI global score, mean (SD) & $5.8(2.3)$ \\
\hline BASFI global score, mean (SD) & $5.5(2.3)$ \\
\hline
\end{tabular}

BASDAI Bath Ankylosing Spondylitis Disease Activity Index, BASFI Bath Ankylosing Spondylitis Functional Index

${ }^{a}$ Patient could select any comorbidities that apply

patients who had received prior treatment, 21 (10.9\%) waited $<1$ week between stopping their previous treatment and initiating secukinumab, $45(23.3 \%)$ waited 1 week to $<1$ month, 35 (18.1\%) waited $1-2$ months, $36(18.7 \%)$ waited 2-3 months, 30 (15.5\%) waited 3-4 months, and 26 $(13.5 \%)$ waited $\geq 5$ months. Among patients with prior treatment, $25.4 \%$ cited lack of efficacy as the main reason for stopping their last treatment; other reasons included side effects (22.8\%), fear of injections/needles (14.5\%), and high co-pay (10.9\%) (Table 2).
Table 2 Treatment history among patients with ankylosing spondylitis (AS) who had been receiving secukinumab for $\geq 3$ months

\begin{tabular}{lc}
\hline & Patients with \\
& AS $(N=200)$ \\
\hline Duration of secukinumab treatment for AS, $n(\%)$ & \\
3-4 months & $33(16.5)$ \\
5-6 months & $39(19.5)$ \\
$7-9$ months & $35(17.5)$ \\
10-12 months & $36(18.0)$ \\
$>12$ months & $57(28.5)$ \\
Approximate time between stopping previous treat- & \\
ment and initiating secukinumab, $n(\%)^{\mathrm{a}}$ & \\
$<1$ week & $21(10.9)$ \\
1 week to 1 month & $45(23.3)$ \\
1 to $<2$ months & $35(18.1)$ \\
2 to $<3$ months & $36(18.7)$ \\
$3-4$ months & $30(15.5)$ \\
$\geq 5$ months & $26(13.5)$ \\
Main reason for stopping previous treatment, $n(\%)^{\mathrm{a}}$ & \\
Lack/loss of efficacy/effectiveness & $49(25.4)$ \\
Unwanted side effects & $44(22.8)$ \\
Anxiety/fear of injections/needles & $28(14.5)$ \\
Co-pay too high & $21(10.9)$ \\
Other reasons ${ }^{\mathrm{b}}$ & $51(26.4)$ \\
Past medication use, $n(\%)$ & $174(87.0)$ \\
Over-the-counter NSAID & $169(84.5)$ \\
Prescription NSAID & $106(53.0)$ \\
Physical therapy & $144(72.0)$ \\
Biologic & $173(86.5)$ \\
Never taken any nonbiologic treatment & \\
Never taken any biologic treatment & \\
\hline
\end{tabular}

NSAID nonsteroidal anti-inflammatory drug

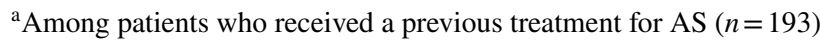

b Other reasons include "insurance would not pay," "treatment was not easy or convenient to take," "injection/infusion was painful," "frequency of dosing was too high," "number of required physician visits was too high," "number of required blood tests was too high," and "other"

\subsection{Ankylosing Spondylitis Symptoms Before and After Secukinumab Initiation}

For all AS symptoms assessed in the online survey, including pain disrupting sleep, fatigue, morning stiffness, pain and stiffness in the lower back or neck, sore areas other than joints, and ankle or heel pain (suggesting enthesitis), the proportion of patients who reported experiencing the symptom after initiating secukinumab was lower than the proportion before secukinumab initiation (Fig. 1a). At the time of survey participation, 72 patients had been receiving secukinumab for 3-6 months, 71 for 7-12 months, 
and 57 for $\geq 12$ months; the frequency of patient-reported symptoms with secukinumab treatment was lower in patients with longer duration of use (Fig. 1b-d). Patients who experienced a little better, moderately better, or much better improvement in AS symptoms since initiating secukinumab were further queried on when they started noticing symptom improvement (Fig. 2). Among patients who reported symptom improvement, $41.9 \%$ reported overall symptom improvement within 4 weeks after secukinumab initiation, $26.4 \%$ within 1-2 months, and $30.4 \%$ after 3 months; a similar trend was observed for each of the individual symptoms. Additionally, most (148 of 200 patients; $74.0 \%$ ) reported overall improvement ("a little [34.5\%]," "moderately [21.0\%]," or "much better [18.5\%]") in AS symptoms since secukinumab initiation compared with before secukinumab initiation; a similar trend was observed for all the individual symptoms analyzed (Fig. 2). Among 86 patients experiencing red and painful eyes, $53(61.7 \%)$ reported improvement in this symptom since secukinumab initiation ("a little better," $16.3 \%$; "moderately better," $22.1 \%$; "much better," $23.3 \%) ; 42$ of these 53 patients (79.2\%) reported symptom improvement within 2 months of initiating secukinumab.

\subsection{Treatment Satisfaction with Secukinumab}

With regard to satisfaction with secukinumab treatment, most patients expressed overall satisfaction ("very," "mostly," or "somewhat satisfied") with secukinumab with regard to symptom improvement (99.0\%), speed of symptom improvement (97.0\%), frequency and method of administration (96.0\% and $91.5 \%$, respectively), ease of use (93.5\%), patient support services $(97.0 \%)$, and side effects, if any (93.0\%) (Fig. 3).

\section{Discussion}

We determined the feasibility of collecting measurable patient-reported experiences with regard to symptom improvement and treatment satisfaction by using a crosssectional study to survey 200 patients with active AS in the USA. This was an exploratory endeavor to determine the practicality of capturing treatment-related experiences using simple questions that resonate with patients. The study design and interview questions will be applied to a large planned prospective study. Here, we evaluated a

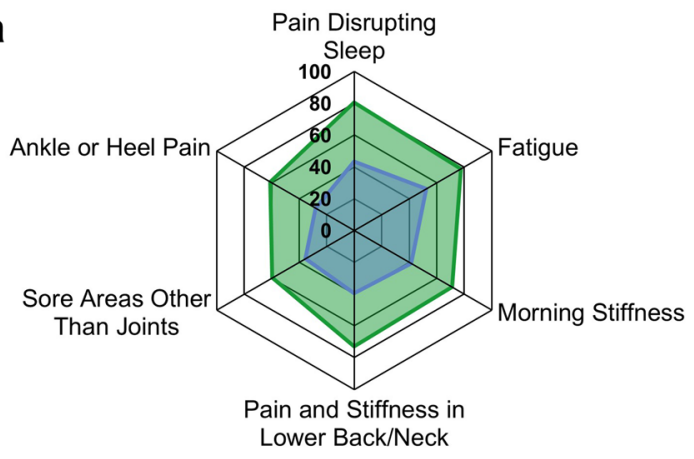

Overall Secukinumab Use $(\mathrm{N}=\mathbf{2 0 0})$

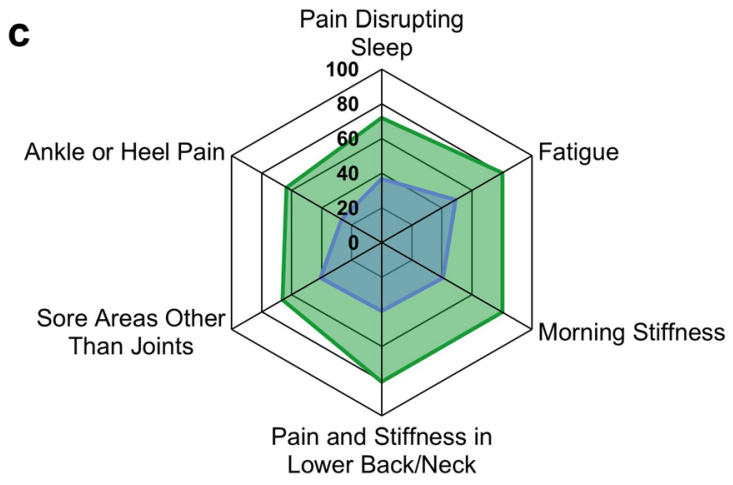

7 to 12 Months of Secukinumab Use $(n=71)$

Fig. 1 Proportion of patients experiencing AS symptoms before and after initiating secukinumab in a the overall population $(N=200)$, b patients with $3-6$ months of secukinumab use $(n=72)$, c patients

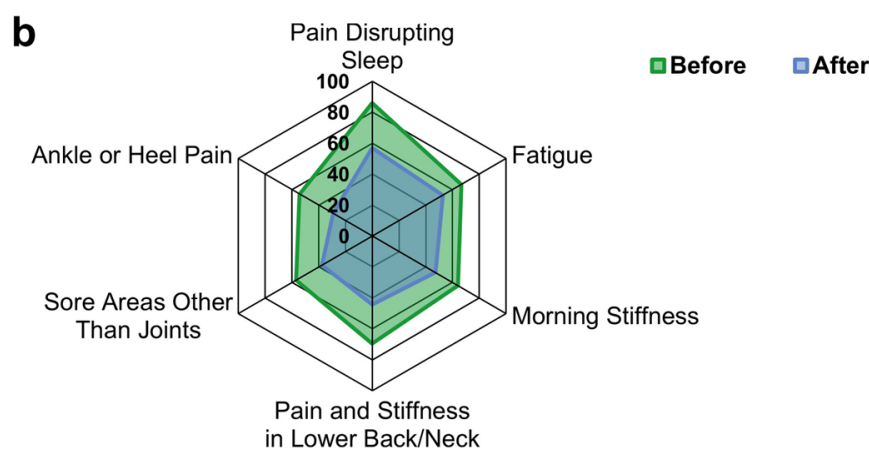

3 to 6 Months of Secukinumab Use $(n=72)$

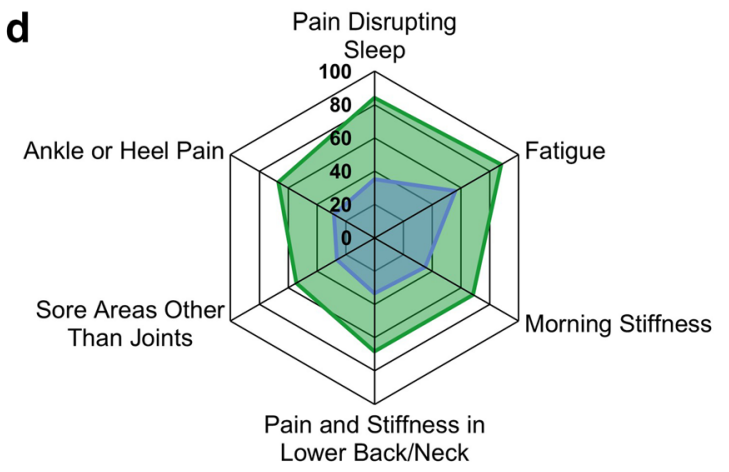

$>12$ Months of Secukinumab Use $(n=57)$

with 7-12 months of secukinumab use $(n=71)$, and d patients with $>12$ months of secukinumab use $(n=57)$. AS ankylosing spondylitis 


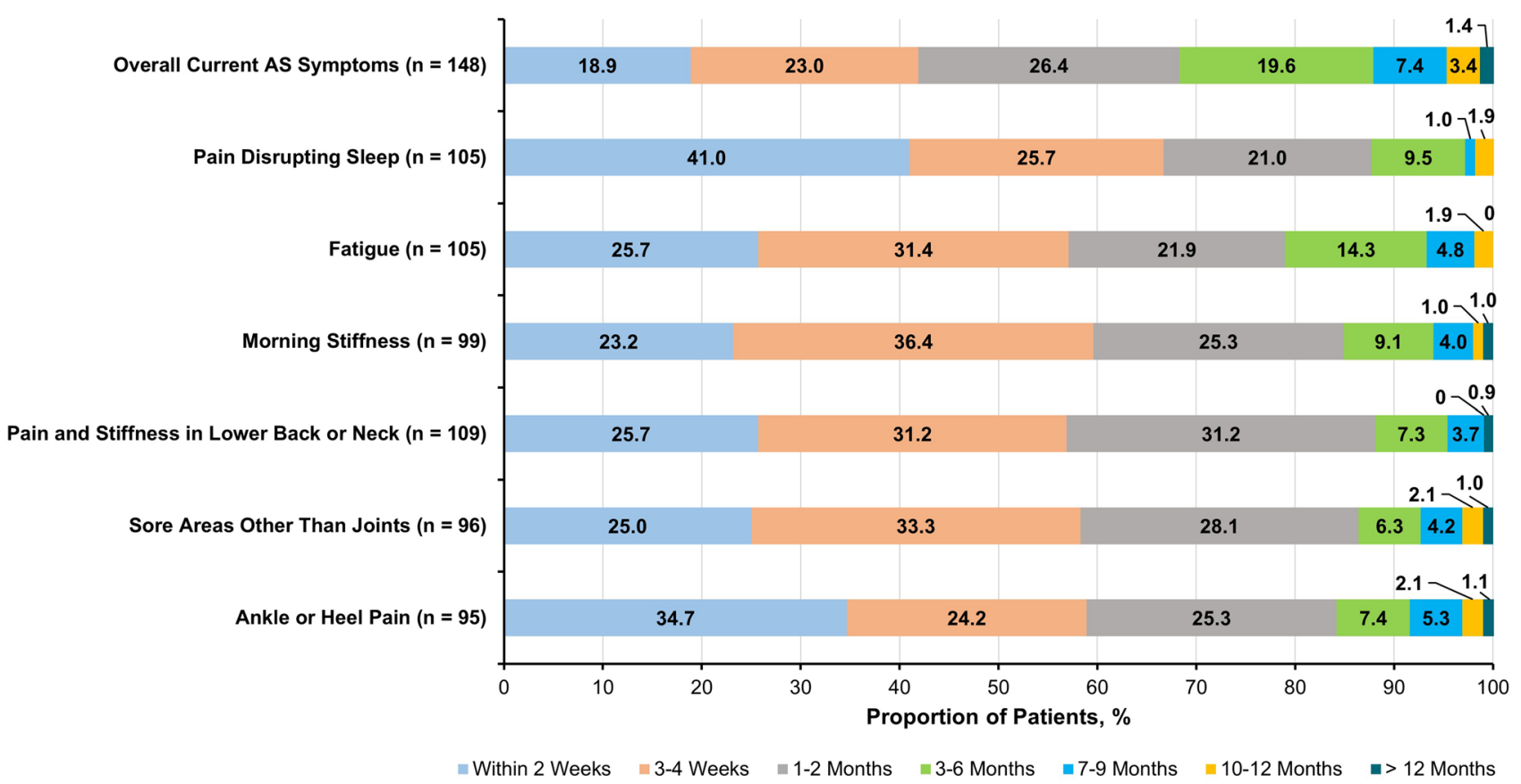

Fig. 2 Patient-reported time to first noticeable improvement in AS symptoms since secukinumab initiation. $A S$ ankylosing spondylitis

patient experience with improvements in various AS symptoms and satisfaction with secukinumab treatment in a real-world setting. The majority of patients in our study reported overall symptom improvement since secukinumab initiation, and almost half of patients surveyed experienced overall improvement within 4 weeks of initiating secukinumab; $>90 \%$ of patients expressed overall satisfaction with their treatment experience.

In AS clinical studies, emphasis is often placed on measuring treatment efficacy, disease activity, and

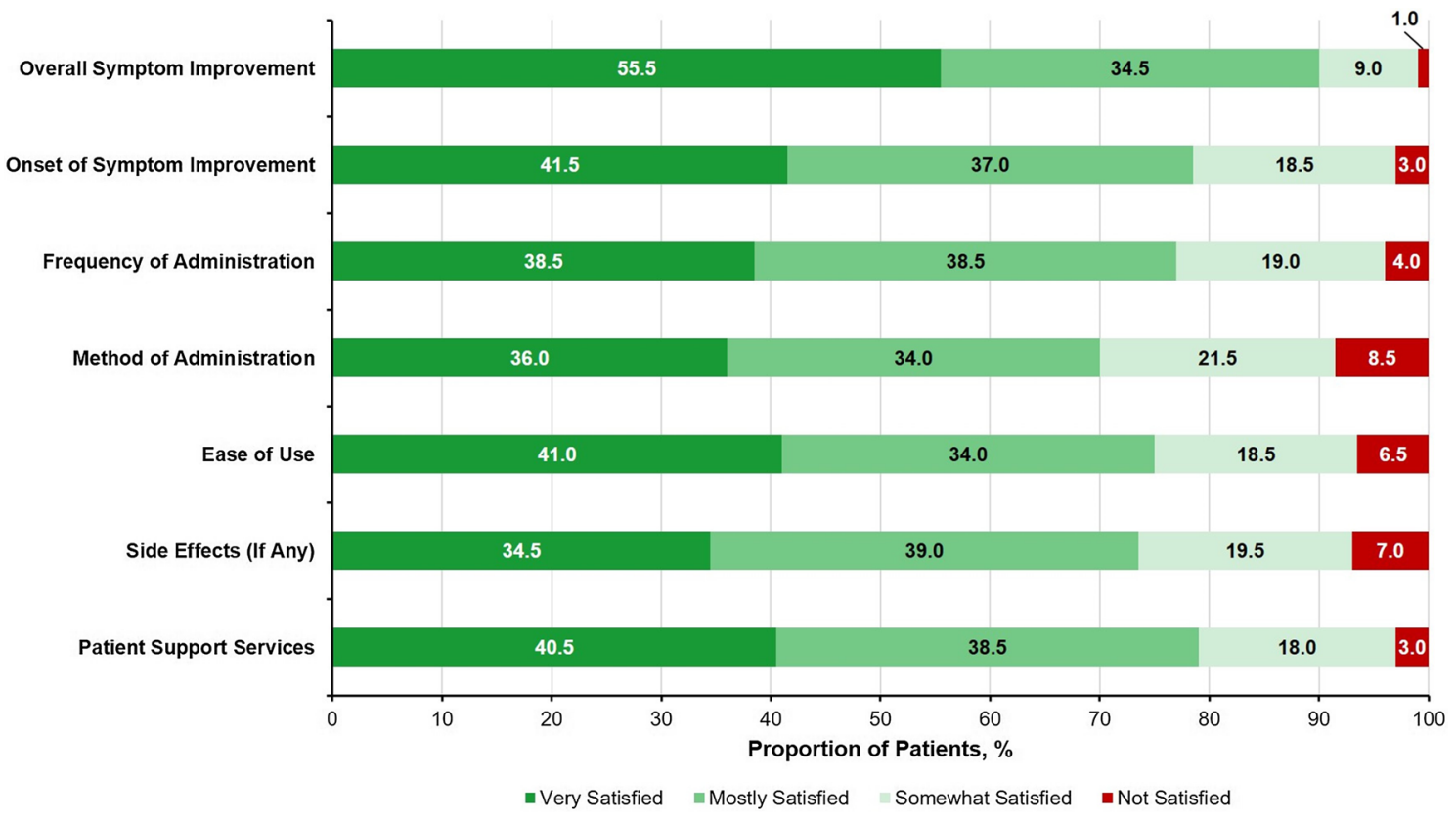

Fig. 3 Overall treatment satisfaction with secukinumab for AS $(n=193)$. AS ankylosing spondylitis 
limitations in physical function. Impairment in QOL, as associated with symptoms such as pain and fatigue [31], and treatment satisfaction are not routinely measured during a clinic visit. A survey of 592 patients with rheumatic diseases, including AS and PsA, indicated that 30-50\% of patients were seldom or never queried on the impact of their disease on QOL, and that normalization of QOL and symptom relief were the most important treatment attributes for these patients [32]. In this study, we sought to understand treatment outcomes and satisfaction as verbalized by the patients. A large proportion of patients reported alleviation of AS symptoms with secukinumab with regard to pain, soreness, and stiffness, which was more pronounced with longer duration of secukinumab use, and overall satisfaction with the speed of symptom improvement, suggesting quicker resolution of these symptoms.

Improvement in pain and fatigue, in particular, is critical in mitigating the physical, social, and psychological aspects of AS [33]. It is important to note that patients often endure other challenges stemming from their disease in addition to disease-specific symptoms. Data obtained from real-world studies can offer invaluable insight into the efficacy and safety of therapeutic agents among more diverse patient populations that are often unrepresented or under-represented in clinical trials with regard to factors such as age, ethnicity, differences in disease severity, and co-morbidities. Additionally, in our real-world study, approximately $87 \%$ of patients surveyed were biologic experienced compared with patients enrolled in clinical trials who are typically biologic naive [34-36]. Few studies have assessed the efficacy and safety of secukinumab in real-world settings [37-40]. Our study is the first to highlight patient-reported experiences with AS symptom improvement and treatment experience with secukinumab among patients with active AS in a US real-world setting, and may complement the efficacy data obtained from clinical trials.

As with all survey-based studies, patient perspectives may be subject to the patients' bias and experience. Physician-confirmed diagnosis and detailed treatment history were not available in this cross-sectional study; in addition, cross-sectional studies are not inherently representative of all patient populations. Furthermore, patient experiences before secukinumab initiation were based on patient recall. No inferences can be made on patient satisfaction and experience with secukinumab compared with specific previous treatments. Because our survey was offered online, only respondents with access to the Internet were able to participate. Our sample size of 200 patients may not be adequately powered for a complete analysis of patient satisfaction, and our patient recruitment method for this study did not allow for the selection of patients who have discontinued treatment with secukinumab. However, our objective was not to evaluate treatment experiences of patients who continued versus those who discontinued treatment; rather, the goal of this survey was to assess the feasibility of collecting patient perspectives on treatment experiences and satisfaction using a self-administered, online survey. A planned future study will examine patients' symptom improvement and experience with secukinumab compared with other biologic therapies as well as patient perspectives among secukinumab responders and nonresponders. Evaluating patient satisfaction is challenging, as "satisfaction" is a complex dynamic - while generic measures of treatment satisfaction exist [41, 42], no rheumatology-specific evaluations are currently available. We relied on perceived contribution of treatment to perceived health improvement, which may not be a correct inference. This study used patient-reported outcomes, such as symptom control and drug tolerability, as measures of satisfaction, and did not include clinical assessment. Additionally, no validated instrument was used to assess the magnitude of patient satisfaction; rather, satisfaction was measured on a 4-point gradient scale (e.g., very satisfied, mostly satisfied, somewhat satisfied, and not satisfied). The study also included some close-ended questions (i.e., questions with "yes" or "no" as answers), which may not adequately capture the nuances of patient experiences.

\section{Conclusion}

Using a web-based survey, the majority of patients with AS in our study who were treated with secukinumab reported improvement in AS symptoms since secukinumab initiation and expressed overall satisfaction with their treatment experience. Additionally, most reported experiencing improvements in their symptoms within a month of secukinumab initiation. Collection of real-world data and patient insights such as these provides perspectives that guide effective and comprehensive clinical decision making. This pilot endeavor indicates that assessment of symptom improvement and treatment satisfaction as conducted in our study is feasible and will be applicable in our future study for evaluation of these elements among responders and nonresponders of AS therapeutic agents.

Acknowledgements Support for third-party writing assistance for this manuscript, furnished by Kheng Bekdache, $\mathrm{PhD}$, of Health Interactions, Inc, was provided by Novartis Pharmaceuticals Corporation, East Hanover, NJ, USA.

\section{Compliance with Ethical Standards}

Funding This study was funded by Novartis Pharmaceuticals Corporation, East Hanover, NJ, USA.

Conflicts of interest M. Magrey served as a consultant for Novartis. M. Bozyczko has nothing to disclose. D. Wolin, M. Mordin, L. McLeod, 
E. Davenport, and C. Chirila are employees of RTI Health Solutions. Y. Park is an employee of Novartis.

Research involving human participants An institutional review board exemption was obtained for the study prior to initiation of data collection.

Informed consent Informed consent was obtained electronically from eligible patients interested in participating in this AS survey.

Open Access This article is distributed under the terms of the Creative Commons Attribution-NonCommercial 4.0 International License (http://creativecommons.org/licenses/by-nc/4.0/), which permits any noncommercial use, distribution, and reproduction in any medium, provided you give appropriate credit to the original author(s) and the source, provide a link to the Creative Commons license, and indicate if changes were made.

\section{References}

1. Dean LE, Jones GT, MacDonald AG, et al. Global prevalence of ankylosing spondylitis. Rheumatology (Oxford). 2014;53:650-7.

2. Dougados M, Baeten D. Spondyloarthritis. Lancet. 2011;377:2127-37.

3. Jang JH, Ward MM, Rucker AN, et al. Ankylosing spondylitis: patterns of radiographic involvement: a re-examination of accepted principles in a cohort of 769 patients. Radiology. 2011;258:192-8

4. Oldroyd J, Schachna L, Buchbinder R, et al. Ankylosing spondylitis patients commencing biologic therapy have high baseline levels of comorbidity: a report from the Australian rheumatology association database. Int J Rheumatol. 2009;2009:268569.

5. Kang JH, Chen YH, Lin HC. Comorbidity profiles among patients with ankylosing spondylitis: a nationwide population-based study. Ann Rheum Dis. 2010;69:1165-8.

6. Bremander A, Petersson IF, Bergman S, et al. Population-based estimates of common comorbidities and cardiovascular disease in ankylosing spondylitis. Arthritis Care Res (Hoboken). 2011;63:550-6.

7. Shen CC, Hu LY, Yang AC, et al. Risk of psychiatric disorders following ankylosing spondylitis: a nationwide population-based retrospective cohort study. J Rheumatol. 2016;43:625-31.

8. Lin $\mathrm{CW}$, Huang YP, Chiu YH, et al. Increased risk of ischemic stroke in young patients with ankylosing spondylitis: a populationbased longitudinal follow-up study. PLoS One. 2014;9:e94027.

9. Han C, Robinson DWJ, Hackett MV, et al. Cardiovascular disease and risk factors in patients with rheumatoid arthritis, psoriatic arthritis, and ankylosing spondylitis. J Rheumatol. 2006;33:2167-72.

10. Boonen A, van der Linden SM. The burden of ankylosing spondylitis. J Rheumatol Suppl. 2006;78:4-11.

11. van der Heijde D, Ramiro S, Landewe R, et al. 2016 update of the ASAS-EULAR management recommendations for axial spondyloarthritis. Ann Rheum Dis. 2017;76:978-91.

12. Ward MM, Deodhar A, Akl EA, et al. American College of Rheumatology/Spondylitis Association of America/Spondyloarthritis Research and Treatment Network 2015 Recommendations for the Treatment of Ankylosing Spondylitis and Nonradiographic Axial Spondyloarthritis. Arthritis Rheumatol. 2016;68:282-98.

13. Maruotti N, d'Onofrio F, Cantatore FP. Metabolic syndrome and chronic arthritis: effects of anti-TNF- $\alpha$ therapy. Clin Exp Med. 2015;15:433-8.
14. Braun J, van den Berg R, Baraliakos X, et al. 2010 update of the ASAS/EULAR recommendations for the management of ankylosing spondylitis. Ann Rheum Dis. 2011;70:896-904.

15. Smith JA, Colbert RA. The interleukin-23/interleukin-17 axis in spondyloarthritis pathogenesis: th17 and beyond. Arthritis Rheumatol. 2014;66:231-41.

16. Xueyi L, Lina C, Zhenbiao W, et al. Levels of circulating Th17 cells and regulatory $\mathrm{T}$ cells in ankylosing spondylitis patients with an inadequate response to anti-TNF- $\alpha$ therapy. J Clin Immunol. 2013;33:151-61.

17. Raychaudhuri SP, Raychaudhuri SK. Mechanistic rationales for targeting interleukin-17A in spondyloarthritis. Arthritis Res Ther. 2017;19:51.

18. Shen H, Goodall JC, Hill Gaston JS. Frequency and phenotype of peripheral blood Th17 cells in ankylosing spondylitis and rheumatoid arthritis. Arthritis Rheum. 2009;60:1647-56.

19. Sherlock JP, Joyce-Shaikh B, Turner SP, et al. IL-23 induces spondyloarthropathy by acting on ROR- $\gamma \mathrm{t}+\mathrm{CD} 3+\mathrm{CD} 4-\mathrm{CD} 8$ - entheseal resident T cells. Nature Med. 2012;18:1069-76.

20. Bowness P, Ridley A, Shaw J, et al. Th17 cells expressing KIR3DL2 + and responsive to HLA-B27 homodimers are increased in ankylosing spondylitis. J Immunol. 2011;186:2672-80.

21. Appel H, Maier R, Wu P, et al. Analysis of IL-17(+) cells in facet joints of patients with spondyloarthritis suggests that the innate immune pathway might be of greater relevance than the Th17-mediated adaptive immune response. Arthritis Res Ther. 2011;13:R95.

22. Kenna TJ, Davidson SI, Duan R, et al. Enrichment of circulating interleukin-17-secreting interleukin-23 receptor-positive $\gamma / \delta$ $\mathrm{T}$ cells in patients with active ankylosing spondylitis. Arthritis Rheum. 2012;64:1420-9.

23. Noordenbos T, Yeremenko N, Gofita I, et al. Interleukin-17-positive mast cell contribute to synovial inflammation in spondylarthritis. Arthritis Rheum. 2012;64:99-109.

24. Cosentyx (secukinumab) [package insert]. East Hanover, NJ: Novartis Pharmaceuticals Corporation 2018.

25. Baeten D, Sieper J, Braun J, et al. Secukinumab, an interleukin-17A Inhibitor, in ankylosing spondylitis. N Engl J Med. 2015;373:2534-48.

26. Pavelka K, Kivitz A, Dokoupilova E, et al. Efficacy, safety, and tolerability of secukinumab in patients with active ankylosing spondylitis: a randomized, double-blind phase 3 study, MEASURE 3. Arthritis Res Ther. 2017;19:285.

27. Lubrano E, Perrotta FM. Secukinumab for ankylosing spondylitis and psoriatic arthritis. Ther Clin Risk Manag. 2016;12:1587-92.

28. Smolen JS, Schols M, Braun J, et al. Treating axial spondyloarthritis and peripheral spondyloarthritis, especially psoriatic arthritis, to target: 2017 update of recommendations by an international task force. Ann Rheum Dis. 2018;77:3-17.

29. Calin A, Garrett S, Whitelock H, et al. A new approach to defining functional ability in ankylosing spondylitis: the development of the Bath Ankylosing Spondylitis Functional Index. J Rheumatol. 1994;21:2281-5.

30. Garrett S, Jenkinson T, Kennedy LG, et al. A new approach to defining disease status in ankylosing spondylitis: the Bath Ankylosing Spondylitis Disease Activity Index. J Rheumatol. 1994;21:2286-91.

31. Dean LE, Macfarlane GJ, Jones GT. Five potentially modifiable factors predict poor quality of life in ankylosing spondylitis: results from the Scotland Registry for Ankylosing Spondylitis. J Rheumatol. 2018;45:62-9.

32. Bolge SC, Eldridge HM, Lofland JH, et al. Patient experience with intravenous biologic therapies for ankylosing spondylitis, Crohn's disease, psoriatic arthritis, psoriasis, rheumatoid arthritis, and ulcerative colitis. Patient Prefer Adherence. 2017;11:661-9. 
33. Brophy S, Davies H, Dennis MS, et al. Fatigue in ankylosing spondylitis: treatment should focus on pain management. Semin Arthritis Rheum. 2013;42:361-7.

34. van der Heijde D, Kivitz A, Schiff MH, et al. Efficacy and safety of adalimumab in patients with ankylosing spondylitis: results of a multicenter, randomized, double-blind, placebo-controlled trial. Arthritis Rheum. 2006;54:2136-46.

35. Inman RD, Davis JC Jr, Heijde D, et al. Efficacy and safety of golimumab in patients with ankylosing spondylitis: results of a randomized, double-blind, placebo-controlled, phase III trial. Arthritis Rheum. 2008;58:3402-12.

36. van der Heijde D, Dijkmans B, Geusens P, et al. Efficacy and safety of infliximab in patients with ankylosing spondylitis: results of a randomized, placebo-controlled trial (ASSERT). Arthritis Rheum. 2005;52:582-91.

37. Georgakopoulos JR, Ighani A, Zhou LL, et al. Efficacy and safety of secukinumab in treating moderate to severe plaque psoriasis in two real-world Canadian dermatology clinics: a multicenter retrospective study. J Eur Acad Dermatol Venereol. 2018;32:e32-4.

38. Momose M, Asahina A, Umezawa Y, et al. Long-term clinical efficacy and safety of secukinumab for Japanese patients with psoriasis: a single-center experience. J Dermatol. 2018;45:318-21.

39. Martinis F, Caimmi C, Carletto A, et al. Real-world efficacy and safety of secukinumab: data from Verona's cohort. Ann Rheum Dis. 2018;77:1544-5.

40. Robertson J, Tindell A, Crosbie Stefan S. Real-world experience of secukinumab for psoriatic arthritis and axial spondyloarthritis. Rheumatology (Oxford). 2018;57(Key075):414.

41. Atkinson MJ, Sinha A, Hass SL, et al. Validation of a general measure of treatment satisfaction, the Treatment Satisfaction Questionnaire for Medication (TSQM), using a national panel study of chronic disease. Health Qual Life Outcomes. 2004;2:12.

42. Ruiz MA, Pardo A, Rejas J, et al. Development and validation of the "TREATMENT Satisfaction with Medicines Questionnaire" (SATMED-Q). Value Health. 2008;11:913-26. 